\title{
Electron pitch-angle diffusion: resonant scattering by waves vs. nonadiabatic effects
}

\author{
A. V. Artemyev ${ }^{1,2}$, K. G. Orlova ${ }^{3,4}$, D. Mourenas ${ }^{5}$, O. V. Agapitov ${ }^{1,6}$, and V. V. Krasnoselskikh ${ }^{1}$ \\ ${ }^{1}$ LPC2E/CNRS - University of Orleans, UMR7328, Orleans, France \\ ${ }^{2}$ Space Research Institute, RAS, Moscow, Russian Federation \\ ${ }^{3}$ Department of Earth and Space Sciences, University of California, Los Angeles, CA, USA \\ ${ }^{4}$ Skobeltsyn Institute of Nuclear Physics, Lomonosov Moscow State University, Moscow, Russian Federation \\ ${ }^{5}$ CEA, DAM, DIF, 91297, Arpajon, France \\ ${ }^{6}$ National Taras Shevchenko University of Kiev, Kiev, Ukraine
}

Correspondence to: A. V. Artemyev (ante0226@gmail.com)

Received: 29 May 2013 - Revised: 2 August 2013 - Accepted: 2 August 2013 - Published: 5 September 2013

\begin{abstract}
In this paper we investigate the electron pitchangle diffusion coefficients in the night-side inner magnetosphere around the geostationary orbit $(L \sim 7)$ due to magnetic field deformation. We compare the effects of resonant wave-particle scattering by lower band chorus waves and the adiabaticity violation of electron motion due to the strong curvature of field lines in the vicinity of the equator. For a realistic magnetic field configuration, the nonadiabatic effects are more important than the wave-particle interactions for high energy ( $>1 \mathrm{MeV}$ ) electrons. For smaller energy, the scattering by waves is more effective than nonadiabatic one. Moreover, the role of nonadiabatic effects increases with particle energy. Therefore, to model electron scattering and transport in the night-side inner magnetosphere, it is important to take into account the peculiarities of high-energy electron dynamics.
\end{abstract}

Keywords. Magnetospheric physics (energetic particles, precipitating) - space plasma physics (charged particle motion and acceleration; wave-particle interactions)

\section{Introduction}

The formation and dynamics of the outer radiation belt in the Earth's magnetosphere is an important but still unsolved problem of plasma physics (see review by Ukhorskiy and Sitnov, 2012, and references therein). The basic processes of electron motion correspond to radial diffusion, adiabatic convection, and resonant wave-particle interactions (see review by Shprits et al., 2008, and references therein). Outside of the plasmapause, electron scattering by whistler and electromagnetic ion cyclotron (EMIC) waves and outward radial diffusion are generally believed to be the most effective mechanisms responsible for rapid electron losses. The former may be expected to prevail during not-too-disturbed periods such that $\mathrm{Kp} \leq 6$, at least for small to medium pitch-angle particles less likely to be affected by magnetopause shadowing (see Kim et al., 2010, and references therein).

Electrons are injected into the inner magnetosphere from the magnetotail region. Such injections can penetrate up to the geostationary orbit (or even deeper; see Dubyagin et al., 2011, and references therein) and bring hot anisotropic electrons and ions to this region. Currents of hot ions substantially modify the local magnetic field configuration (Daglis et al., 1999). Therefore, electron scattering in this region is essentially different from the scattering in the classical dipole magnetic field configuration (Orlova and Shprits, 2010; Ma et al., 2012). However, not only the wave-particle interactions are influenced by the magnetic field configuration, but also the dynamics of electrons can also be substantially modified due to the change of the magnetic field line geometry.

The classical theory of radiation belts assumes the conservation of three invariants of electron motion (Ukhorskiy and Sitnov, 2012, and references therein). The first invariant (magnetic moment) corresponds to fast electron gyrorotation around the field line. Conservation of this invariant allows the use of the guiding center approximation. However, in contrast to the exact invariants of motion, adiabatic invariants 

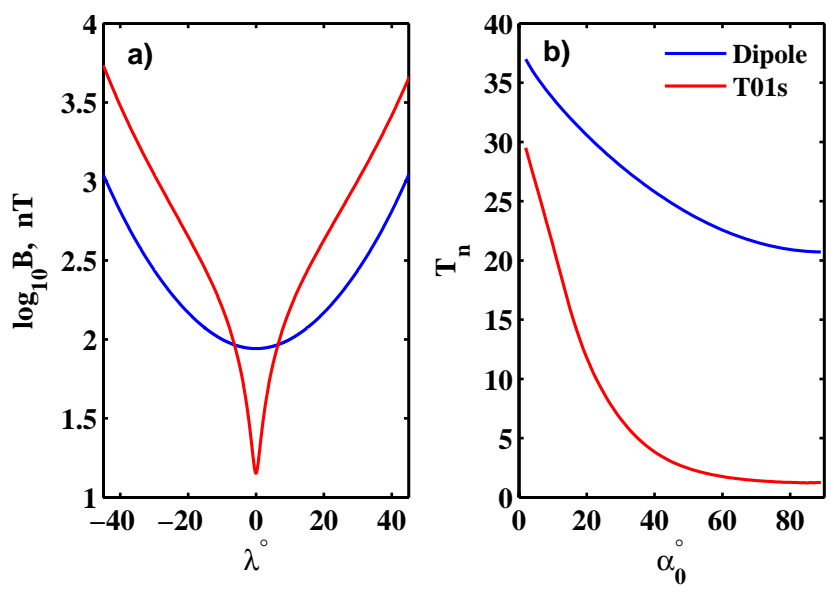

Fig. 1. Panels (a) and (b) show the dependence of the magnetic field amplitude on magnetic latitude and electron normalized bounce pe$\operatorname{riod} T_{\mathrm{n}}$ as a function of equatorial pitch angle, respectively, for the dipole and T01s field models.

are conserved with a certain (finite) accuracy (Landau and Lifshitz, 1988). This accuracy is controlled by so-called adiabaticity parameters. For example, the conservation of the first adiabatic invariant is determined by the small parameter $\varepsilon=\rho_{0} / R_{\mathrm{c}}$, where $\rho_{0}$ is electron gyroradius at the equator and $R_{\mathrm{c}}$ is the curvature radius of magnetic field lines at the equator (Chirikov, 1979). There is a jump of magnetic moment every time an electron crosses the magnetic equator. Equations for these jumps were derived for various magnetic field geometries (Birmingham, 1984; Il'ina et al., 1993; Delcourt et al., 1994). In the dipole magnetic field configuration, $\varepsilon$ is small enough to neglect such jumps. However, realistic geomagnetic field lines become more and more stretched on the night side as distance increases and may have a relatively small curvature radius in the vicinity of the magnetic equator. Thus, the scattering of electrons due to adiabaticity violation should be revised for this region. In this paper we compare the effects of electron pitch-angle diffusion due to nonadiabatic effects and due to resonant wave-particle interactions in a realistic magnetic field around the geostationary orbit.

\section{Typical spatial scales and timescales}

Global statistics (Le et al., 2004; Borovsky and Denton, 2010) and empirical models (e.g., Sitnov et al., 2008) show that during disturbed geomagnetic conditions the magnitude of the equatorial magnetic field can be decreased down to $\sim 10 \mathrm{nT}$ around the geostationary orbit $(L \sim 6-7)$. Also, a thin current sheet is formed (Dubyagin et al., 2013, and references therein) in this region with a spatial scale along the dawn-dusk direction of about $\sim 2-5 R_{\mathrm{E}}$.

As a realistic geomagnetic field, we use an external T01s model (Tsyganenko et al., 2003) plus an internal International Geomagnetic Reference Field (IGRF) model (referred to further in the text as T01s model) during the disturbed conditions for Dst $=-100 \mathrm{nT}$ (see details of other model parameters in Orlova et al., 2012). Figure 1a shows the magnetic field amplitude $B$ as a function of magnetic latitude $\lambda$ in the T01s and dipole fields. There is a rapid nondipolar decrease of $B$ in the vicinity of the equator. Such a modification of the magnetic field influences electron motion.

We calculate the normalized bounce period $T_{\mathrm{n}}$ of electron motion (divided by the Earth's radius and multiplied by electron's velocity) as a function of equatorial pitch angle $\alpha_{0}$ for the T01s model at $L=7$, MLT $=0$, for the disturbed conditions and compare it with the dipole approximation $4 L T\left(\alpha_{0}\right)$, where $T\left(\alpha_{0}\right) \approx 1.3809-0.1851\left(\sin \alpha_{0}\right)^{1 / 2}-$ $0.4559\left(\sin \alpha_{0}\right)^{0.863}$ (Orlova and Shprits, 2011). The deformation of the field line geometry results in a substantial modification of the bounce period, which decreases for large pitchangle electrons (see Fig. 1b).

The azimuthal drift of electrons is also influenced by the magnetic field configuration. The azimuthal drift velocity can be increased by up to $100-200 \%$ around the geostationary orbit in case of strong magnetic field deviation from the dipole model (Vapirev and Jordanova, 2007). It is caused by the gradient increase $d B / d \lambda$ in the vicinity of the equator (azimuthal drift is the sum of gradient and curvature drifts $\sim d B / d \lambda$; see Newkirk and Walt (1964)). The period of electron azimuthal drift in the dipole magnetic field at $L \sim 6$ 7 varies from $T_{\mathrm{D}} \sim 0.3 \times 10^{3} \mathrm{~s}$ to $T_{\mathrm{D}} \sim 3 \times 10^{3} \mathrm{~s}$ for energy range $[0.1,3] \mathrm{MeV}$ (Ukhorskiy and Sitnov, 2012). Therefore, $1 \mathrm{MeV}$ electrons spend around $\Delta t \sim 100-200 \mathrm{~s}$ in the perturbed region with nondipolar magnetic field. The increase of the azimuthal drift should reduce this time to $\Delta t \sim 50-100 \mathrm{~s}$ (here we assume that the region with substantially stretched field lines covers 2-4h of MLT (see Le et al., 2004; Dubyagin et al., 2013)).

\section{Pitch-angle diffusion}

To compute the bounce-averaged pitch-angle diffusion coefficients $\left\langle D_{\alpha_{0} \alpha_{0}}\right\rangle$ corresponding to resonant wave-particle interactions and nonadiabatic effects, we use equations developed by Orlova et al. (2012) and Young et al. (2008), respectively. We consider that electrons are scattered by lower band chorus waves, which are the most intense whistlermode emissions in the inner magnetosphere (see Meredith et al., 2012; Agapitov et al., 2013, and references therein). $\left\langle D_{\alpha_{0} \alpha_{0}}\right\rangle$ for the resonant wave-particle interactions is obtained by using the quasi-linear theory (Glauert and Horne, 2005, see also references therein) with a wave-frequency distribution $\hat{B}^{2}(\omega)=K \exp \left(-\left(\omega_{\mathrm{m}}-\omega\right)^{2} / \delta \omega^{2}\right)$, where $K$ is a normalization coefficient and parameters $\omega_{\mathrm{m}}$ and $\delta \omega$ are the frequency of the maximum wave power and the bandwidth, respectively, taken from Orlova et al. (2012). Normal angles of wave propagation $\theta$ are assumed to be distributed as $\sim \exp \left(-X^{2} / X_{\mathrm{w}}^{2}\right)$, where $X=\tan \theta$ and $X_{\mathrm{w}}=\tan \left(30^{\circ}\right)$. 
The averaging procedure over electron bounce oscillations is described in Orlova and Shprits (2011). We consider chorus wave amplitudes of $100 \mathrm{pT}$, in agreement with Cluster and CRRES measurements at $L \sim 7$ during disturbed periods (Shprits et al., 2007; Agapitov et al., 2013). For $\left\langle D_{\alpha_{0} \alpha_{0}}\right\rangle$ calculation, we use the T01s magnetic field model presented in Fig. 1a.

To obtain $\left\langle D_{\alpha_{0} \alpha_{0}}\right\rangle$ due to nonadiabatic effects, we take into account jumps of electron magnetic moment. Since pitchangle scattering does not change the particle energy, we can use the normalized magnetic moment $\mu=(1 / 2) \sin ^{2} \alpha_{0}$ dependent on equatorial pitch angle only. $\mu$ experiences a jump $\delta \mu$ every time an electron crosses the equatorial plane (Delcourt et al., 1994): $\delta \mu=A_{0} \cos \phi+A_{1}$, where $\phi$ is the electron gyrophase and parameters $A_{0}$ and $A_{1}$ depend on the magnetic field topology. $A_{0}$ is given by (Young et al., 2002)

$A_{0}=e^{c(\varepsilon)}\left(\zeta_{1}^{a_{1}(\varepsilon)} \zeta_{2}^{a_{2}(\varepsilon)}+C(\varepsilon)\right) \frac{\sin \left(\Lambda(\varepsilon) \alpha_{0}\right) \cos ^{\beta(\varepsilon)} \alpha_{0}}{\sin \left(\Lambda(\varepsilon) \alpha_{\max }\right) \cos ^{\beta(\varepsilon)} \alpha_{\max }}$.

Coefficients $\zeta_{1,2}$ depend on magnetic field configuration and are defined as

$\zeta_{1}=\left.R_{\mathrm{c}} \frac{\partial^{2} R_{\mathrm{c}}}{\partial s^{2}}\right|_{s=0}, \zeta_{2}=\left.\frac{R_{\mathrm{c}}^{2}}{B_{0}} \frac{\partial^{2} B}{\partial s^{2}}\right|_{s=0}$,

where $B(s)$ and $R_{\mathrm{c}}(s)$ denote magnetic field amplitude and curvature radius as a function of the arc length along the field line $s$, respectively. Coefficients $C(\varepsilon), c(\varepsilon), a_{1}(\varepsilon), a_{2}(\varepsilon)$, $\Lambda(\varepsilon), \beta(\varepsilon)$, and constant $\alpha_{\max }$ do not depend on magnetic field configuration and can be found in Young et al. (2002). The pitch-angle diffusion coefficient is obtained by averaging over the ensemble of jumps $\delta \mu$ (Young et al., 2008):

$\left\langle D_{\alpha_{0} \alpha_{0}}\right\rangle=A_{0}^{2} /\left(T\left(\alpha_{0}\right) \sin ^{2} \alpha_{0} \cos ^{2} \alpha_{0}\right)$.

Empirical models may underestimate the scale of magnetic field deformations in the vicinity of the equator (see discussion in Dubyagin et al., 2013). They also do not allow varying this scale without changing other system parameters. As shown by Orlova et al. (2012), for electrons with equatorial pitch angles $\sim 70-90^{\circ}$, the bounce-averaged pitch-angle scattering rates due to wave-particle interaction are not sensitive to the magnetic field deformation. These electrons resonantly interact with waves in the close vicinity of the equator. Conversely, for electrons with equatorial pitch angles smaller than about $20^{\circ}$ and energies $\geq 0.5 \mathrm{MeV}$ at $L \sim 7$, cyclotron resonance occurs only at latitudes above $10^{\circ}$. Thus, $\left\langle D_{\alpha_{0} \alpha_{0}}\right\rangle$ related to wave-particle interaction can be accurately calculated without modifying the T01s model to describe better the fine structure of the magnetic field in the close vicinity of the equator.

In contrast to resonant wave-particle interaction, nonadiabatic scattering is very sensitive to the fine structure of the magnetic field at the equator and is mainly determined by equatorial magnetic field and corresponding derivatives.
Thus, it is principally important to describe accurately the magnetic field configuration at the equator for the calculation of $\left\langle D_{\alpha_{0} \alpha_{0}}\right\rangle$ related to nonadiabatic effects.

Therefore, to improve upon the empirical model we further assume that the magnetic field can be approximated by a simple model of current sheet with $B_{z}=\sigma B_{0 x}$ and $B_{x}=$ $B_{0 x} \tanh \left(z / L_{\mathrm{CS}}\right)$ in the vicinity of the equator. We choose the near-equatorial region at midnight when GSM $x$ axis almost coincides with the radial direction. $B_{0 x}$ is the field value at the boundary of the considered region with stretched field lines (i.e., $B_{0 x} \sim 100 \mathrm{nT}$ ), $\sigma$ a constant parameter defining the strength of magnetic field perturbation, $z$ the coordinate along the normal to the equatorial plane, and $L_{\mathrm{CS}}$ the current sheet thickness. For $z=0$ we get

$$
\frac{\partial^{2} R_{c}}{\partial s^{2}}=\frac{3+2 \sigma^{2}}{\sigma L_{\mathrm{CS}}}, \quad \frac{\partial^{2} B}{\partial s^{2}}=\frac{B_{0 x}}{\sigma L_{\mathrm{CS}}^{2}}
$$

and $\zeta_{1}=3+2 \sigma^{2}, \zeta_{2}=\sigma$ where curvature radius at the equator is $R_{\mathrm{c}}=L_{\mathrm{CS}} \sigma$. We assume $\sigma \sim 0.15$, which is typical for $L \sim 7$ for Dst $=-100 \mathrm{nT}$. The thickness of the current sheet $L_{\mathrm{CS}}$ is about 1.0-2 $R_{\mathrm{E}}$ (Le et al., 2004; Dubyagin et al., 2013).

We compare $\left\langle D_{\alpha_{0} \alpha_{0}}\right\rangle$ for resonant wave-particle interactions with those computed using Eq. (1) (see Fig. 2). The role of nonadiabatic effects in electron scattering increases with energy, while scattering rates due to wave-particle interactions decrease with energy. As a result, the most important part of pitch-angle diffusion for high-energy electrons $(\geq 1 \mathrm{MeV})$ is provided by nonadiabatic scattering (for $\left.L_{\mathrm{CS}} \leq 1.5 R_{\mathrm{E}}\right)$.

It is also worthwhile to compare directly the magnetic field models used for the calculations of $\left\langle D_{\alpha_{0} \alpha_{0}}\right\rangle$ due to wave-particle interaction and nonadiabatic effects. Let us assume that the T01s model correctly describes the magnetic field configuration in the vicinity of the equator. For a $\tanh \left(z / L_{\mathrm{CS}}\right)$ field shape at $z<L_{\mathrm{CS}}, L_{\mathrm{CS}}$ in the T01s model corresponds to 5-8 degrees in latitude (at $L \sim 7$ ) and, thus, to $\sim 0.7-1 R_{\mathrm{E}}$. With stretching of the magnetic field, the pitchangle scattering rates due to wave-particle interactions increase at small to moderate pitch angles (Orlova and Shprits, 2010; Orlova et al., 2012). Therefore, in the mentioned pitch-angle range and for $L_{\mathrm{CS}}>1 R_{\mathrm{E}}$, these diffusion coefficients can only be smaller than those presented in Fig. 2 for the T01s model. Thus, the wave-particle scattering rates in Fig. 2 at $\alpha_{0}<45^{\circ}$, which mainly determine the crucial electron lifetime (e.g., see Orlova and Shprits, 2010; Artemyev et al., 2013), are the maximum ones for $L_{\mathrm{CS}} \geq 1 R_{\mathrm{E}}$. At equatorial pitch angles larger than $70^{\circ}$, pitch-angle scattering rates due to wave-particle interaction are almost independent of the magnetic field deformation (Orlova et al., 2012). 

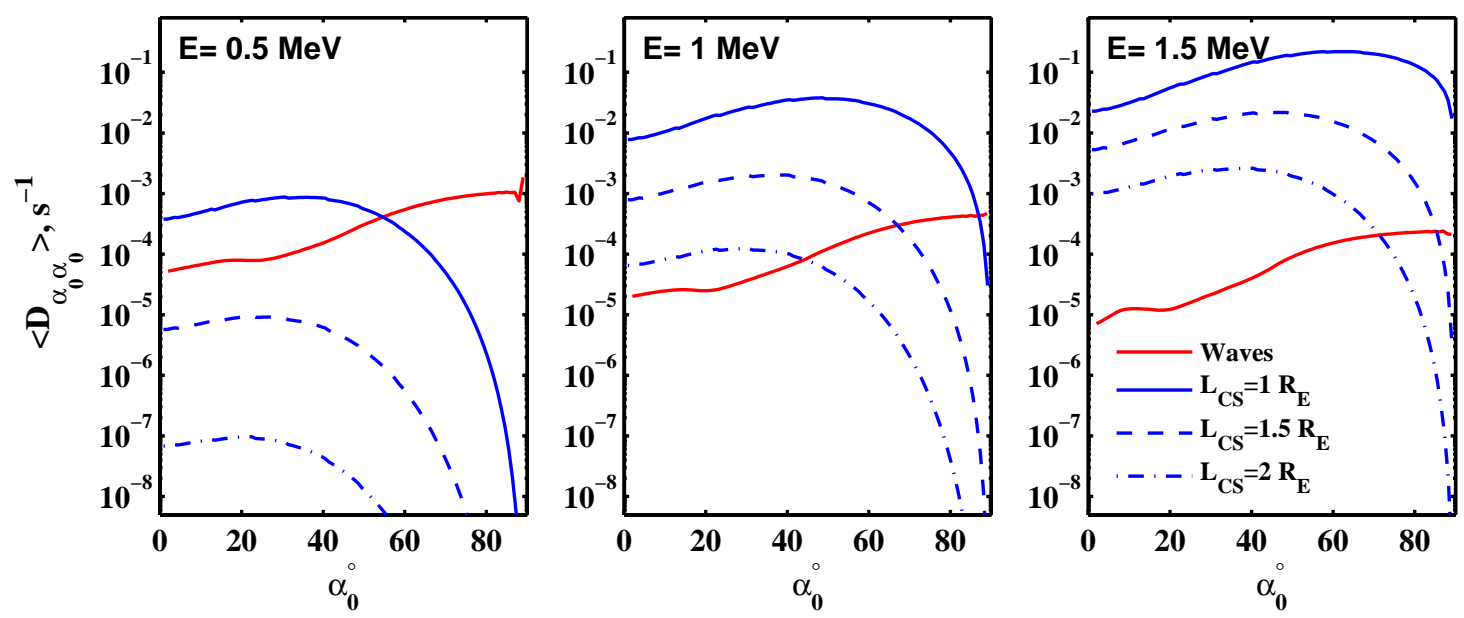

Fig. 2. Comparison of the pitch-angle diffusion coefficients for resonant wave-particle interactions and for nonadiabatic effects.

\section{Conclusions}

We show that the modification of the magnetic field configuration on the night side around the geostationary orbit results in an intensification of electron scattering due to nonadiabatic electron motion in the vicinity of the equator. Corresponding diffusion coefficients increase with electron energy in contrast with diffusion induced by resonant wave-particle interactions. As a result, for high energy electrons $(\geq 1 \mathrm{MeV})$ nonadiabaticity of electron motion is more important than effects of wave-particle interactions.

However, we should mention several mechanisms that may increase the efficiency of scattering by waves. First of all, we have only considered quasi-parallel wave propagation (i.e., $\langle\theta\rangle=0$ ). Due to fast damping of oblique waves at $L \sim 7$ induced by hot electrons (Chen et al., 2013), this approach seems to be reasonable. Nevertheless, a certain portion of oblique chorus waves can still be present even at $L \sim 7$ (Agapitov et al., 2013) and, therefore, substantially increase the pitch-angle diffusion coefficients (Artemyev et al., 2013). The second possibility is to take into account other wave emissions in this region, e.g., magnetosonic waves (see comparison of magnetosonic and chorus waves impacts on electron scattering in Mourenas et al., 2013). The nightside region around $L \sim 7$ is also filled by intense, coherent whistler wave packets brought on or induced by injections. These waves have large enough amplitudes to interact with particles in the nonlinear regime and, as a result, may intensify pitch-angle diffusion (see review by Shklyar and Matsumoto, 2009, and references therein). Although all these effects may modify the pitch-angle diffusion coefficients, we have considered here the most general scattering by lower band chorus waves at $L \sim 7$ using measured amplitudes during disturbed periods.

In the dipolar field, the effective lifetime for $1.5 \mathrm{MeV}$ electrons due to nonadiabatic effects $\left(\tau_{\text {nad }} \sim\left(T_{\mathrm{D}} / \Delta t\right)\left\langle D_{\alpha \alpha}\left(\alpha_{\mathrm{LC}}\right)\right\rangle^{-1}\right.$ with loss-cone angle $\alpha_{\mathrm{LC}} \sim 2-3^{\circ}$ ) is more than one day at $L \sim 7$ on the night side. We show that in the realistic field, it decreases to $\tau_{\text {nad }} \sim 1-6 \mathrm{~h}$. Nevertheless, this reduced lifetime still remains substantially larger than the time that particles actually spend in the region with significantly nondipolar field configuration $\left(\Delta t \sim 10^{2} \mathrm{~s}\right)$. Thus, we do not expect that nonadiabatic effects can totally scatter electrons into the loss cone immediately after injections. A significant part of the injected electron population leaves the region of the nondipole field configuration before being scattered. However, the obtained decrease of lifetimes by one order of magnitude should correspond to some intensification of energetic electron precipitation in the region of initial injections, independent of the level of wave activity (see discussion of isotropic precipitation boundary in Dubyagin et al., 2013, and references therein). Moreover, diffusion rates due to nonadiabatic effects for electrons with the energies $\geq 1 \mathrm{MeV}$ can substantially exceed the strong diffusion limit (Kennel and Petschek, 1966), which is about $4 \times 10^{-4} \mathrm{~s}^{-1}$ for these energies at $L \sim 7$. Thus, nonadiabatic scattering can support stable electron precipitation in the region with strongly deformed magnetic field.

Acknowledgements. We are thankful to Y. Y. Shprits for valuable discussion of obtained results. The work of A. V. Artemyev, O. V. Agapitov, and V. V. Krasnoselskikh was supported by CNES through the grant "Modele d'ondes." The research of K. G. Orlova was supported by the NASA Living With a Star Jack Eddy Postdoctoral Fellowship Program, administered by the University Corporation for Atmospheric Research. The work of A. V. Artemyev and V. V. Krasnoselskikh is also partially supported by the Ministry of Education and Science of Russian Federation, project 8527.

Topical Editor L. Blomberg thanks one anonymous referee for her/his help in evaluating this paper. 


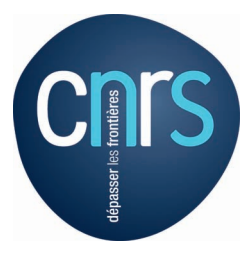

The publication of this article is financed by CNRS-INSU.

\section{References}

Agapitov, O., Artemyev, A., Krasnoselskikh, V., Khotyaintsev, Y. V., Mourenas, D., Breuillard, H., Balikhin, M., and Rolland, G.: Statistics of Whistler-Mode Waves in the Outer Radiation Belt: Cluster STAFF-SA measurements, J. Geophys. Res., 118, 34073420, doi:10.1002/jgra.50312, 2013.

Artemyev, A. V., Mourenas, D., Agapitov, O. V., and Krasnoselskikh, V. V.: Parametric validations of analytical lifetime estimates for radiation belt electron diffusion by whistler waves, Ann. Geophys., 31, 599-624, doi:10.5194/angeo-31-599-2013, 2013.

Birmingham, T. J.: Pitch angle diffusion in the Jovian magnetodisc, J. Geophys. Res., 89, 2699-2707, doi:10.1029/JA089iA05p02699, 1984.

Borovsky, J. E. and Denton, M. H.: Magnetic field at geosynchronous orbit during high-speed stream-driven storms: Connections to the solar wind, the plasma sheet, and the outer electron radiation belt, J. Geophys. Res., 115, A08217, doi:10.1029/2009JA015116, 2010.

Chen, L., Thorne, R. M., Li, W., and Bortnik, J.: Modeling the Wave Normal Distribution of Chorus Waves, J. Geophys. Res.: Space Physics, 118, 1074-1088, doi:10.1029/2012JA018343, 2013.

Chirikov, B. V.: A universal instability of many-dimensional oscillator systems, Physics Reports, 52, 263-379, doi:10.1016/03701573(79)90023-1, 1979.

Daglis, I. A., Thorne, R. M., Baumjohann, W., and Orsini, S.: The terrestrial ring current: Origin, formation, and decay, Rev. Geophys., 37, 407-438, doi:10.1029/1999RG900009, 1999.

Delcourt, D. C., Martin Jr., R. F., and Alem, F.: A simple model of magnetic moment scattering in a field reversal, Geophys. Res. Lett., 21, 1543-1546, doi:10.1029/94GL01291, 1994.

Dubyagin, S., Sergeev, V., Apatenkov, S., Angelopoulos, V., Runov, A., Nakamura, R., Baumjohann, W., McFadden, J., and Larson, D.: Can flow bursts penetrate into the inner magnetosphere?, Geophys. Res. Lett., 38, L08102, doi:10.1029/2011GL047016, 2011.

Dubyagin, S., Ganushkina, N., Apatenkov, S., Kubyshkina, M., Singer, H., and Liemohn, M.: Geometry of duskside equatorial current during magnetic storm main phase as deduced from magnetospheric and low-altitude observations, Ann. Geophys., 31, 395-408, doi:10.5194/angeo-31-395-2013, 2013.

Glauert, S. A. and Horne, R. B.: Calculation of pitch angle and energy diffusion coefficients with the PADIE code, J. Geophys. Res., 110, A04206, doi:10.1029/2004JA010851, 2005.

Il'ina, A. N., Il'in, V. D., Kuznetsov, S. N., Yushkov, B. Y., Amirkhanov, I. V., and Il'In, I. V.: Model of nonadiabatic charged-particle motion in the field of a magnetic dipole, Soviet JETP, 77, 246-252, 1993.

Kennel, C. F. and Petschek, H. E.: Limit on Stably Trapped Particle Fluxes, J. Geophys. Res., 71, 1-28, doi:10.1029/2009JA014523,
1966.

Kim, K. C., Lee, D.-Y., Kim, H.-J., Lee, E. S., and Choi, C. R.: Numerical estimates of drift loss and Dst effect for outer radiation belt relativistic electrons with arbitrary pitch angle, J. Geophys. Res., 115, A03208, doi:10.1029/2009JA014523, 2010.

Landau, L. D. and Lifshitz, E. M.: Vol. 1: Mechanics, Course of Theoretical Physics, Oxford: Pergamon, 1988.

Le, G., Russell, C. T., and Takahashi, K.: Morphology of the ring current derived from magnetic field observations, Ann. Geophys., 22, 1267-1295, doi:10.5194/angeo-22-1267-2004, 2004.

Ma, Q., Ni, B., Tao, X., and Thorne, R. M.: Evolution of the plasma sheet electron pitch angle distribution by whistler-mode chorus waves in non-dipole magnetic fields, Ann. Geophys., 30, 751760, doi:10.5194/angeo-30-751-2012, 2012.

Meredith, N. P., Horne, R. B., Sicard-Piet, A., Boscher, D., Yearby, K. H., Li, W., and Thorne, R. M.: Global model of lower band and upper band chorus from multiple satellite observations, J. Geophys. Res., 117, A10225, doi:10.1029/2012JA017978, 2012.

Mourenas, D., Artemyev, A. V., Agapitov, O. V., and Krasnoselskikh, V. V.: Analytical estimates of electron quasi-linear diffusion by fast magnetosonic waves, J. Geophys. Res., 118, 30963112, doi:10.1002/jgra.50349, 2013, 2013.

Newkirk, L. L. and Walt, M.: Longitudinal Drift Velocity of Geomagnetically Trapped Particles, J. Geophys. Res., 69, 17591763, doi:10.1029/JZ069i009p01759, 1964.

Orlova, K. G. and Shprits, Y. Y.: Dependence of pitch-angle scattering rates and loss timescales on the magnetic field model, Geophys. Res. Lett., 37, L05105, doi:10.1029/2009GL041639, 2010.

Orlova, K. G. and Shprits, Y. Y.: On the bounce-averaging of scattering rates and the calculation of bounce period, Phys. Plasmas, 18, 092904, doi:10.1063/1.3638137, 2011.

Orlova, K. G., Shprits, Y. Y., and Ni, B.: Bounce-averaged diffusion coefficients due to resonant interaction of the outer radiation belt electrons with oblique chorus waves computed in a realistic magnetic field model, J. Geophys. Res., 117, A07209, doi:10.1029/2012JA017591, 2012.

Shklyar, D. and Matsumoto, H.: Oblique Whistler-Mode Waves in the Inhomogeneous Magnetospheric Plasma: Resonant Interactions with Energetic Charged Particles, Surv. Geophys., 30, 55104, doi:10.1007/s10712-009-9061-7, 2009.

Shprits, Y. Y., Meredith, N. P., and Thorne, R. M.: Parameterization of radiation belt electron loss timescales due to interactions with chorus waves, Geophys. Res. Lett., 34, L11110, doi:10.1029/2006GL029050, 2007.

Shprits, Y. Y., Subbotin, D. A., Meredith, N. P., and Elkington, S. R.: Review of modeling of losses and sources of relativistic electrons in the outer radiation belt II: Local acceleration and loss, J. Atmos. Solar-Terr. Phys., 70, 1694-1713, doi:10.1016/j.jastp.2008.06.014, 2008.

Sitnov, M. I., Tsyganenko, N. A., Ukhorskiy, A. Y., and Brandt, P. C.: Dynamical data-based modeling of the storm-time geomagnetic field with enhanced spatial resolution, J. Geophys. Res., 113, A07218, doi:10.1029/2007JA013003, 2008.

Tsyganenko, N. A., Singer, H. J., and Kasper, J. C.: Storm-time distortion of the inner magnetosphere: How severe can it get?, J. Geophys. Res., 108, 1209, doi:10.1029/2002JA009808, 2003.

Ukhorskiy, A. Y. and Sitnov, M. I.: Dynamics of Radiation Belt Particles, Space Sci. Rev., doi:10.1007/s11214-012-9938-5, 2012. 
Vapirev, A. E. and Jordanova, V. K.: Calculation of bounceaveraged velocities and hydrogen densities for a stormtime magnetic field, Geophys. Res. Lett., 34, L10103, doi:10.1029/2007GL029380, 2007.

Young, S. L., Denton, R. E., Anderson, B. J., and Hudson, M. K.: Empirical model for $\mu$ scattering caused by field line curvature in a realistic magnetosphere, J. Geophys. Res., 107, 1069, doi:10.1029/2000JA000294, 2002.
Young, S. L., Denton, R. E., Anderson, B. J., and Hudson, M. K.: Magnetic field line curvature induced pitch angle diffusion in the inner magnetosphere, J. Geophys. Res., 113, 3210, doi:10.1029/2006JA012133, 2008. 